\title{
ON A PROBLEM OF MATSUMOTO AND TAMAGAWA CONCERNING MONODROMIC FULLNESS OF HYPERBOLIC CURVES: GENUS ZERO CASE
}

\author{
YUICHIRO HOSHI
}

(Received August 31, 2011)

\begin{abstract}
In the present paper, we discuss a problem concerning monodromic fullness of hyperbolic curves over number fields posed by Matsumoto and Tamagawa in the case where a given hyperbolic curve is of genus zero.
\end{abstract}

Introduction. In the present paper, we discuss a problem concerning monodromic fullness of hyperbolic curves over number fields posed by Matsumoto and Tamagawa in the case where a given hyperbolic curve is of genus zero. First, let us review the notion of monodromic fullness: Let $(g, r)$ be a pair of nonnegative integers such that $2 g-2+r>0$ and $k$ a number field (i.e., a finite extension of the field of rational numbers). Write $\mathfrak{P r i m} \mathfrak{s}$ for the set of all prime numbers, $\mathcal{M}_{g,[r]}$ for the moduli stack of hyperbolic curves (cf. "Curves" in "Notation and Conventions") of type ( $g, r)$ over $k$, and $\mathcal{M}_{g, r}$ for the moduli stack of ordered $r$-pointed proper smooth curves of genus $g$ over $k$. Thus, by forgetting the order of marked points, we have a natural finite étale Galois covering $\mathcal{M}_{g, r} \rightarrow \mathcal{M}_{g,[r]}$ whose Galois group is isomorphic to the symmetric group $\mathfrak{S}_{r}$ on $r$ letters; in particular, we have a normal open subgroup $\pi_{1}\left(\mathcal{M}_{g, r}\right) \subseteq \pi_{1}\left(\mathcal{M}_{g,[r]}\right)$ such that the quotient $\pi_{1}\left(\mathcal{M}_{g,[r]}\right) / \pi_{1}\left(\mathcal{M}_{g, r}\right)$ is isomorphic to $\mathfrak{S}_{r}$. Let $X$ be a hyperbolic curve of type $(g, r)$ over $k$ and $k$ an algebraic closure of $k$. Then, for each $l \in \mathfrak{P r i m e s}$, if we write $\pi_{1}^{\{l\}}\left(X \otimes_{k} \bar{k}\right)$ for the maximal pro-l quotient of $\pi_{1}\left(X \otimes_{k} \bar{k}\right)$, then we have two natural outer representations on $\pi_{1}^{\{l\}}\left(X \otimes_{k} \bar{k}\right)$

$$
\rho_{X / k}^{\{l\}}: G_{k} \stackrel{\text { def }}{=} \operatorname{Gal}(\bar{k} / k) \longrightarrow \operatorname{Out}\left(\pi_{1}^{\{l\}}\left(X \otimes_{k} \bar{k}\right)\right),
$$

i.e., the pro- $l$ outer Galois representation associated to the hyperbolic curve $X / k$, and

$$
\rho_{g,[r]}^{\{l\}}: \pi_{1}\left(\mathcal{M}_{g,[r]}\right) \longrightarrow \operatorname{Out}\left(\pi_{1}^{\{l\}}\left(X \otimes_{k} \bar{k}\right)\right)
$$

i.e., the pro- $l$ outer universal monodromy representation. Since $\rho_{X / k}^{\{l\}}$ factors, via the outer homomorphism $G_{k} \rightarrow \pi_{1}\left(\mathcal{M}_{g,[r]}\right)$ induced by the classifying morphism Spec $k \rightarrow \mathcal{M}_{g,[r]}$ of $X / k$, through $\rho_{g,[r]}^{\{l\}}$, we obtain natural inclusions

$$
\rho_{X / k}^{\{l\}}\left(G_{k}\right) \subseteq \rho_{g,[r]}^{\{l\}}\left(\pi_{1}\left(\mathcal{M}_{g,[r]}\right)\right) \supseteq \rho_{g,[r]}^{\{l\}}\left(\pi_{1}\left(\mathcal{M}_{g, r}\right)\right) .
$$

2000 Mathematics Subject Classification. Primary 14H30.

Key words and phrases. Monodromic fullness, hyperbolic curve, number field.

Partly supported by the Grant-in-Aid for Young Scientists (B), No.20740010, the Ministry of Education, Culture, Sports, Science and Technology, Japan. 
For a nonempty subset $\Sigma \subseteq \mathfrak{P r i m e s}$, we shall say that $X$ is $\Sigma$-monodromically full (over $k$ ) if, for each $l \in \Sigma$, it holds that $\rho_{g,[r]}^{\{l\}}\left(\pi_{1}\left(\mathcal{M}_{g, r}\right)\right) \subseteq \rho_{X / k}^{\{l\}}\left(G_{k}\right)$ (cf. [2, Definition 2.2, (i)]); $X$ is quasi- $\Sigma$-monodromically full (over $k$ ) if, for each $l \in \Sigma$, it holds that $\rho_{X / k}^{\{l\}}\left(G_{k}\right)$ is open in $\rho_{g,[r]}^{\{l\}}\left(\pi_{1}\left(\mathcal{M}_{g,[r]}\right)\right)$ (cf. [2, Definition 2.2, (iii)]). In [4], Matsumoto and Tamagawa proved that, for each $l \in \mathfrak{P r i m e s}$, there are many hyperbolic curves over number fields which are $l$-monodromically full (cf. [4, Theorem 1.2]).

Now let us recall that if $l \in \mathfrak{P r i m e s}$, and $E$ is an elliptic curve over $k$, whose $l$-adic Tate module we denote by $T_{l}(E)$, then we have a natural isomorphism $\pi_{1}^{\{l\}}\left(E \otimes_{k} \bar{k}\right) \stackrel{\sim}{\rightarrow} T_{l}(E)$ and a noncanonical isomorphism $T_{l}(E) \stackrel{\sim}{\rightarrow} Z_{l}^{\oplus 2}$, that determine isomorphisms $\operatorname{Out}\left(\pi_{1}^{\{l\}}\left(E \otimes_{k}\right.\right.$ $\bar{k})) \stackrel{\sim}{\rightarrow} \operatorname{Aut}\left(T_{l}(E)\right) \stackrel{\sim}{\rightarrow} \mathrm{GL}_{2}\left(\boldsymbol{Z}_{l}\right)$. Moreover, the image of the étale fundamental group of the moduli stack of elliptic curves over $\bar{k}$ by the $l$-adic universal monodromy representation coincides, with respect to the isomorphisms $\operatorname{Out}\left(\pi_{1}^{\{l\}}\left(E \otimes_{k} \bar{k}\right)\right) \stackrel{\sim}{\rightarrow} \operatorname{Aut}\left(T_{l}(E)\right) \stackrel{\sim}{\rightarrow} \mathrm{GL}_{2}\left(\boldsymbol{Z}_{l}\right)$, with $\mathrm{SL}_{2}\left(\boldsymbol{Z}_{l}\right) \subseteq \mathrm{GL}_{2}\left(\boldsymbol{Z}_{l}\right)$. Furthermore, in [6], Serre proved the following result concerning the image of the pro- $l$ Galois representation associated to $E / k$.

THEOREM (cf. [6]). In the notation of the above discussion, the following four conditions are equivalent:

(0) E does not admit complex multiplication over $\bar{k}$.

(1) For any $l \in \mathfrak{P r i m e s}$, the image of the l-adic Galois representation $G_{k} \rightarrow$ $\operatorname{Aut}\left(T_{l}(E)\right)$ associated to $E / k$ is an open subgroup of $\operatorname{Aut}\left(T_{l}(E)\right)$.

(2) There exists an $l \in \mathfrak{P r i m e s}$ such that the l-adic Galois representation $G_{k} \rightarrow$ $\operatorname{Aut}\left(T_{l}(E)\right)$ associated to $E / k$ is surjective.

(3) There exists a finite subset $\Sigma$ of $\mathfrak{P r i m e s}$ such that if $l \notin \Sigma$, then the l-adic Galois representation $G_{k} \rightarrow \operatorname{Aut}\left(T_{l}(E)\right)$ associated to $E / k$ is surjective.

From this point of view, "the property of being (quasi-)monodromically full" may be regarded as an analogue for hyperbolic curves of "the property of not admitting complex multiplication" for elliptic curves.

As an analogue for hyperbolic curves of the equivalences “(1) $\Leftrightarrow(2) \Leftrightarrow$ (3)” in the above result due to Serre, in [4], Matsumoto and Tamagawa posed the following problem concerning monodromic fullness of hyperbolic curves over number fields.

Problem (cf. [4, Problem 4.1]). Let X be a hyperbolic curve over a number field. Then are the following three conditions equivalent?

$\left(\mathrm{MT}_{1}\right) X$ is quasi-Primes-monodromically full.

$\left(\mathrm{MT}_{2}\right)$ There exists an $l \in \mathfrak{P r i m e s}$ such that $X$ is $l$-monodromically full.

$\left(\mathrm{MT}_{3}\right)$ There exists a finite subset $\Sigma$ of $\mathfrak{P r i m e s}$ such that $X$ is $(\mathfrak{P r i m e s} \backslash \Sigma)$-monodromically full.

In the present paper, we discuss the above problem due to Matsumoto and Tamagawa in the case where the given hyperbolic curve $X$ is of genus 0 . More concretely, we prove the following two results. 
THEOREM A. Let $k$ be a number field. Then there exists a split (cf. "Curves" in "Notation and Conventions") hyperbolic curve of type $(0,4)$ over $k$ which satisfies $\left(\mathrm{MT}_{3}\right)$, hence also $\left(\mathrm{MT}_{2}\right)$, but does not satisfy $\left(\mathrm{MT}_{1}\right)$. Moreover, for any positive integer $r>4$, there exists a split hyperbolic curve of type $(0, r)$ over $k$ which satisfies $\left(\mathrm{MT}_{2}\right)$ but does not satisfy $\left(\mathrm{MT}_{1}\right)$.

THEOREM B. Let $k$ be a number field, $k_{0} \subseteq k$ a subfield of $k$, and $X$ a hyperbolic curve of type $(0,4)$ over $k_{0}$ such that $X \otimes_{k_{0}} k$ is split. Thus, one verifies easily that there exists $\lambda \in k \backslash\{0,1\}$ such that the hyperbolic curve $X \otimes_{k_{0}} k$ is isomorphic to $\boldsymbol{P}_{k}^{1} \backslash\{0,1, \lambda, \infty\}$ over $k$. Here, we note that the set

$$
\mathfrak{m}_{X} \stackrel{\text { def }}{=}\left\{\lambda, \frac{1}{\lambda}, 1-\lambda, \frac{1}{1-\lambda}, \frac{\lambda}{\lambda-1}, \frac{\lambda-1}{\lambda}\right\} \subseteq k
$$

(cf. [2, Definition 7.10]) depends only on (and completely determines!) the isomorphism class of the hyperbolic curve $X \otimes_{k_{0}} k$ over $k$. Consider the following five conditions:

(1) The above set $\mathfrak{m}_{X}$ does not contain any unit of the ring of integers of $k$.

(2) There exists a finite subset $\Sigma$ of $\mathfrak{P r i m e s}$ such that $X$ is $(\mathfrak{P r i m e s} \backslash \Sigma)$-monodromically full (over $k_{0}$ ) (cf. [2, Definition 2.2, (i)]).

(3) There exists an $l \in \mathfrak{P r i m e s}$ such that $X$ is $l$-monodromically full (over $k_{0}$ ).

(4) There exists an $l \in \mathfrak{P r i m e s}$ such that $X$ is quasi-l-monodromically full (over $k_{0}$ ) (cf. [2, Definition 2.2, (iii)]).

(5) The above set $\mathfrak{m}_{X}$ does not contain any root of unity of the ring of integers of $k$.

Then we have implications

$$
(1) \Longrightarrow(2) \Longrightarrow(3) \Longrightarrow(4) \Longrightarrow(5) \text {. }
$$

If, moreover, $k$ is quadratic imaginary, then we have equivalences

$$
(1) \Longleftrightarrow(2) \Longleftrightarrow(3) \Longleftrightarrow(4) \Longleftrightarrow(5) \text {. }
$$

In particular, if $k$ is quadratic imaginary, then the equivalence " $\left(\mathrm{MT}_{2}\right) \Leftrightarrow\left(\mathrm{MT}_{3}\right)$ " for such an $X$ holds.

The author would like to thank Makoto Matsumoto and Akio Tamagawa for inspiring me by means of their problem given in [4]. The author also would like to thank the referee for some comments and, especially, a suggestion concerning the statement of Theorem B.

Notation And Conventions. Numbers: The notation Primes will be used to denote the set of all prime numbers. The notation $\boldsymbol{Z}$ will be used to denote the ring of rational integers. If $p$ is a prime number, then the notation $\boldsymbol{F}_{p}$ will be used to denote the finite field with $p$ elements and the notation $\boldsymbol{Z}_{p}$ will be used to denote the $p$-adic completion of $\boldsymbol{Z}$. We shall refer to a finite extension of the field of rational numbers as a number field.

Profinite groups: If $G$ is a profinite group, then we shall write $\operatorname{Aut}(G)$ for the group of (continuous) automorphisms of $G, \operatorname{Inn}(G) \subseteq \operatorname{Aut}(G)$ for the group of inner automorphisms of $G$, and

$$
\operatorname{Out}(G) \stackrel{\text { def }}{=} \operatorname{Aut}(G) / \operatorname{Inn}(G) \text {. }
$$


If, moreover, $G$ is topologically finitely generated, then one verifies easily that the topology of $G$ admits a basis of characteristic open subgroups, which thus induces a profinite topology on the group $\operatorname{Aut}(G)$, hence also a profinite topology on the group $\operatorname{Out}(G)$.

If $G$ and $H$ are profinite groups, then we shall write $\operatorname{Hom}(G, H)$ for the set of (continuous) homomorphisms from $G$ to $H$. Then the group $\operatorname{Inn}(H)$ naturally acts on the set $\operatorname{Hom}(G, H)$. We shall refer to an element of the quotient set $\operatorname{Hom}(G, H) / \operatorname{Inn}(H)$ as an outer homomorphism from $G$ to $H$.

If $G$ is a profinite group, then we shall write $G^{\mathrm{ab}}$ for the abelianization of $G$, i.e., the quotient of $G$ by the normal closed subgroup generated by the commutators of $G$.

Curves: Let $k$ be a field and $X$ a scheme over $k$. For a pair $(g, r)$ of nonnegative integers, we shall say that $X$ is a smooth curve of type $(g, r)$ over $k$ if there exist a scheme $X^{\mathrm{cpt}}$ of dimension 1 which is smooth, proper, and geometrically connected over $k$ and a closed subscheme $D \subseteq X^{\mathrm{cpt}}$ of $X^{\mathrm{cpt}}$ which is étale and of degree $r$ over $k$ such that the complement of $D$ in $X^{\mathrm{cpt}}$ is isomorphic to $X$ over $k$, and, moreover, a geometric fiber of $X^{\mathrm{cpt}} \rightarrow$ Spec $k$ is (a necessarily smooth, proper, and connected curve) of genus $g$. Note that it follows immediately that if $X$ is a smooth curve of type $(g, r)$ over $k$, then the pair " $\left(X^{\mathrm{cpt}}, D\right)$ " is uniquely determined up to canonical isomorphism. We shall say that $X$ is a hyperbolic curve over $k$ if there exists a pair $(g, r)$ of nonnegative integers such that $2 g-2+r>0$, and, moreover, $X$ is a smooth curve of type ( $g, r)$ over $k$. We shall say that $X$ is a tripod over $k$ if $X$ is a smooth curve of type $(0,3)$ over $k$. (Thus, any tripod over $k$ is a hyperbolic curve over $k$.) Suppose that there exists a pair $(g, r)$ of nonnegative integers such that $X$ is a smooth curve of type $(g, r)$ over $k$. Then we shall say that $X$ is split if " $D$ " appearing in the definition of the term "smooth curve of type $(g, r)$ " is isomorphic to the disjoint union of $r$ copies of Spec $k$ over $k$.

1. Various objects arising from split tripods. In this section, let us recall various objects arising from split tripods. Let $k$ be a field of characteristic 0 and $\bar{k}$ an algebraic closure of $k$. Write $G_{k} \stackrel{\text { def }}{=} \mathrm{Gal}(\bar{k} / k)$ for the absolute Galois group of $k$ determined by the algebraic closure $\bar{k}$ and

$$
\mathcal{M} \stackrel{\text { def }}{=} \boldsymbol{P}_{k}^{1} \backslash\{0,1, \infty\}=\operatorname{Spec} k\left[t^{ \pm 1}, 1 /(1-t)\right],
$$

where $t$ is an indeterminate, for the split tripod over $k$. Now we have a natural identification

$$
\mathcal{M}(k) \simeq k \backslash\{0,1\}
$$

and an exact sequence of profinite groups

$$
1 \longrightarrow \pi_{1}\left(\mathcal{M} \otimes_{k} \bar{k}\right) \longrightarrow \pi_{1}(\mathcal{M}) \longrightarrow G_{k} \longrightarrow 1 .
$$

Moreover, for each prime number $l$, write

$$
\mu_{l} \subseteq \bar{k}^{\times}
$$

for the subgroup of $\bar{k}^{\times}$of all $l$-powers roots of unity. 
DEFINITION 1.1. Let $l$ be a prime number.

(i) We shall write

for the maximal pro-l quotient of $\pi_{1}\left(\mathcal{M} \otimes_{k} \bar{k}\right)$.

(ii) Since the closed subgroup $\pi_{1}\left(\mathcal{M} \otimes_{k} \bar{k}\right)$ of $\pi_{1}(\mathcal{M})$ is normal, conjugation by elements of $\pi_{1}(\mathcal{M})$ naturally determines continuous homomorphisms

$$
\pi_{1}(\mathcal{M}) \longrightarrow \operatorname{Aut}\left(\Delta^{\{l\}}\right) ; G_{k} \longrightarrow \operatorname{Out}\left(\Delta^{\{l\}}\right),
$$

where we refer to the discussion entitled "Profinite Groups" in "Notation and Conventions" concerning the profinite topologies of $\operatorname{Aut}\left(\Delta^{\{l\}}\right)$ and $\operatorname{Out}\left(\Delta^{\{l\}}\right)$. We shall write

$$
\widetilde{\rho}^{\{l\}} ; \rho^{\{l\}}
$$

for the above continuous homomorphisms, respectively. It follows immediately from the various definitions involved that these homomorphisms fit into the following commutative diagram of profinite groups

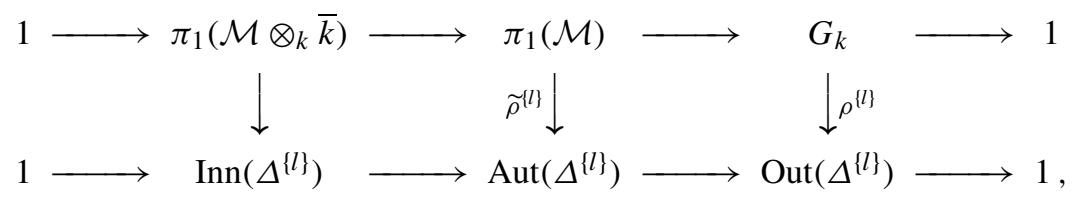

where the horizontal sequences are exact; moreover, since $\Delta^{\{l\}}$ is center-free, the left-hand vertical arrow factors as the composite of the natural surjection $\pi_{1}\left(\mathcal{M} \otimes_{k} \bar{k}\right) \rightarrow \Delta^{\{l\}}$ and the

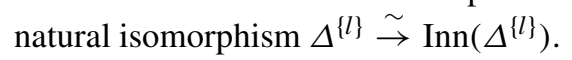

(iii) We shall write

$$
\pi_{1}(\mathcal{M}) \rightarrow \Phi^{\{l\}} \quad\left(\text { resp. } G_{k} \rightarrow G_{k}^{\mathrm{tpd}-l}\right)
$$

for the quotient of $\pi_{1}(\mathcal{M})$ (resp. $G_{k}$ ) by the kernel of the homomorphism $\widetilde{\rho}^{\{l\}}$ (resp. $\rho^{\{l\}}$ ). Thus, the commutative diagram in (ii) determines an exact sequence of profinite groups

$$
1 \longrightarrow \Delta^{\{l\}} \longrightarrow \Phi^{\{l\}} \longrightarrow G_{k}^{\mathrm{tpd}-l} \longrightarrow 1
$$

(iv) We shall write

$$
k^{\mathrm{tpd}-l}(\subseteq \bar{k})
$$

for the Galois extension of $k$ corresponding to the quotient $G_{k} \rightarrow G_{k}^{\text {tpd- } l}$, i.e., $G_{k}^{\text {tpd- } l}=$ $\operatorname{Gal}\left(k^{\mathrm{tpd}-l} / k\right)$.

(v) Let $\lambda \in k$ be such that $\lambda \neq 0,1$. Then we shall write

$$
s_{\lambda}: G_{k} \longrightarrow \pi_{1}(\mathcal{M})
$$

for the outer homomorphism induced by $\lambda \in k \backslash\{0,1\} \simeq \mathcal{M}(k)$.

REMARK 1.2. In [3], the notation $\Delta_{\mathcal{M} / k}^{\{l\}}$ (resp. $\widetilde{\rho}_{\mathcal{M} / k}^{\{l\}} ; \rho_{\mathcal{M} / k}^{\{l\}} ; \Phi_{\mathcal{M} / k}^{\{l\}} ; \Gamma_{\mathcal{M} / k}^{\{l\}}$ ) was used to denote the object $\Delta^{\{l\}}$ (resp. $\widetilde{\rho}^{\{l\}} ; \rho^{\{l\}} ; \Phi^{\{l\}} ; G_{k}^{\text {tpd-l }}$ ) defined in Definition 1.1 of the present paper (cf. [3, Definition 1]). 
LEMma 1.3. Let $l$ be a prime number and $\lambda \in k \backslash\{0,1\}$. Then the following three conditions are equivalent:

(1) The split hyperbolic curve of type $(0,4)$ over $k$

$$
\boldsymbol{P}_{k}^{1} \backslash\{0,1, \lambda, \infty\}
$$

is l-monodromically full (resp. quasi-l-monodromically full) (cf. [2, Definition 2.2]).

(2) The $k$-rational point of $\mathcal{M}$ naturally corresponding to $\lambda \in k \backslash\{0,1\}$ is l-monodromically full (resp. quasi-l-monodromically full) (cf. [3, Definition 8, (i)] in the case where we take " $(X, n)$ " to be $(\mathcal{M}, 1))$.

(3) The image of the composite

$$
G_{k} \stackrel{s_{\lambda}}{\longrightarrow} \pi_{1}(\mathcal{M}) \longrightarrow \Phi^{\{l\}}
$$

is $\Phi^{\{l\}}$ (resp. is an open subgroup of $\Phi^{\{l\}}$ ).

PROOF. The equivalence "(1) $\Leftrightarrow(2)$ ” follows from the equivalence in [3, Remark 11, (ii)] in the case where we take " $(X, n)$ " to be $(\mathcal{M}, 1)$. The equivalence " $(2) \Leftrightarrow(3)$ " follows from [3, Proposition 19, (iv)] in the case where we take " $(X, m)$ " to be $(\mathcal{M}, 1)$.

2. Monodromic fullness for hyperbolic curves of type $(0,4)$. In the present section, we discuss the monodromic fullness for hyperbolic curves of type $(0,4)$. We maintain the notation of the preseding $\S 1$.

DEFINITION 2.1 (cf. [1]). Let $l$ be an odd prime number.

(i) We shall write

$$
S_{l}
$$

for the minimal set of finite subsets of $\boldsymbol{P} \frac{1}{k}(\bar{k}) \simeq \bar{k} \cup\{\infty\}$ which satisfies the following three conditions: (1) $\{0,1, \infty\} \in S_{l}$. (2) If $S \in S_{l}$, then $\left\{a \in \bar{k} ; a^{l} \in S\right\} \cup\{\infty\} \in S_{l}$. (3) If $S \in \boldsymbol{S}_{l}$, and $\phi$ is an automorphism of $\boldsymbol{P}_{\bar{k}}^{1}$ over $\bar{k}$ such that $\{0,1, \infty\} \subseteq \phi(S)$, then $\phi(S) \in \boldsymbol{S}_{l}$.

(ii) We shall write

$$
\boldsymbol{E}_{l} \subseteq \bar{k}^{\times}
$$

for the subgroup of $\bar{k}^{\times}$generated by the elements of $S \backslash\{0, \infty\}$ for all $S \in S_{l}$.

Some of main results of [1] are as follows.

Proposition 2.2. Let l be an odd prime number. Then the following hold:

(i) $k^{\text {tpd-l }}=k\left(\boldsymbol{E}_{l}\right)$.

(ii) $\left(\boldsymbol{E}_{l}\right)^{l}=\boldsymbol{E}_{l}$.

(iii) $\mu_{l} \subseteq \boldsymbol{E}_{l}$.

(iv) Suppose that $k$ is a number field. Then the algebraic extension $k^{\mathrm{tpd}-l} / k$ is unramified at any nonarchimedean prime of $k$ whose residue characteristic is not equal to $l$.

Proof. This follows from [1, Theorems A, B]. 
REMARK 2.3. Even if $l=2$, by considering the composite

$$
G_{k} \stackrel{\rho^{\{l\}}}{\longrightarrow} \operatorname{Out}\left(\Delta^{\{l\}}\right) \longrightarrow \operatorname{Aut}\left(\left(\Delta^{\{l\}}\right)^{\mathrm{ab}}\right),
$$

one verifies easily that $\mu_{l^{\infty}} \subseteq k^{\mathrm{tpd}-l}$.

LEMMA 2.4. Let $l$ be an odd prime number. Then $l \in \boldsymbol{E}_{l}$.

Proof. It follows from the condition (1) of Definition 2.1, (i), that $\{0,1, \infty\} \in S_{l}$. Thus, it follows from the condition (2) of Definition 2.1, (i), that

$$
S \stackrel{\text { def }}{=}\left\{0,1, \zeta_{l}, \zeta_{l}^{2}, \ldots, \zeta_{l}^{l-1}, \infty\right\} \in S_{l},
$$

where $\zeta_{l} \in \bar{k}$ is a primitive $l$-th root of unity. Now since the automorphism $\phi$ of $\boldsymbol{P}_{\bar{k}}^{1}$ over $\bar{k}$ given by " $t \mapsto 1-t$ " satisfies that $\{0,1, \infty\} \subseteq \phi(S)$, it follows from the condition (3) of Definition 2.1, (i), that

$$
\phi(S)=\left\{1,0,1-\zeta_{l}, 1-\zeta_{l}^{2}, \ldots, 1-\zeta_{l}^{l-1}, \infty\right\} \in S_{l} .
$$

Therefore,

$$
l=\prod_{i=1}^{l-1}\left(1-\zeta_{l}^{i}\right) \in \boldsymbol{E}_{l} .
$$

This completes the proof of Lemma 2.4.

LeMma 2.5. Let $l$ be a prime number. Suppose that $\mu_{l^{\infty}} \subseteq k$. For each positive integer $n$, write

$$
C_{l^{n}} \stackrel{\text { def }}{=} \operatorname{Spec} k\left[x^{ \pm 1}, y^{ \pm 1}\right] /\left(x^{l^{n}}+y^{l^{n}}-1\right) \longrightarrow \mathcal{M},
$$

where $x$ and $y$ are indeterminates, for the finite étale Galois $\left(\boldsymbol{Z} / l^{n} \boldsymbol{Z}\right)^{\oplus 2}$-covering of $\mathcal{M}$ given by “ $t \mapsto x^{l^{n}}$ ”. Write, moreover,

$$
\pi_{1}(\mathcal{M}) \rightarrow Q_{l} \simeq Z_{l}^{\oplus 2}
$$

for the quotient of $\pi_{1}(\mathcal{M})$ determined by the $C_{l^{n}}$ 's. Then the following hold:

(i) Let $n$ be a positive integer and $\lambda \in k \backslash\{0,1\} \simeq \mathcal{M}(k)$. Write $k_{n}(\subseteq \bar{k})$ for the finite extension of $k$ corresponding to the quotient of $G_{k}$ determined by the composite

$$
G_{k} \stackrel{s_{\lambda}}{\rightarrow} \pi_{1}(\mathcal{M}) \rightarrow Q_{l} \rightarrow Q_{l} / l^{n} Q_{l}
$$

Then we have an equality

$$
k_{n}=k\left(\lambda^{1 / l^{n}},(1-\lambda)^{1 / l^{n}}\right) .
$$

(ii) The quotient $\pi_{1}(\mathcal{M}) \rightarrow Q_{l}$ factors through the quotient $\pi_{1}(\mathcal{M}) \rightarrow \Phi^{\{l\}}$.

PROOF. First, we verify the assertion (i). It follows immediately from the various definitions involved that the fiber of the finite étale Galois covering $C_{l^{n}} \rightarrow \mathcal{M}$ at $\lambda \in k \backslash\{0,1\} \simeq$ $\mathcal{M}(k)$ is isomorphic to the disjoint union of finitely many copies of Spec $k_{n}$. Thus, the assertion (i) follows immediately from the explicit description of the finite étale covering $C_{l^{n}} \rightarrow \mathcal{M}$. This completes the proof of the assertion (i). 
Next, we verify the assertion (ii). To verify the assertion (ii), it is immediate that it suffices to verify the fact that for any positive integer $n$, the quotient $\pi_{1}(\mathcal{M}) \rightarrow\left(\boldsymbol{Z} / l^{n} \boldsymbol{Z}\right)^{\oplus 2}$ determined by the finite étale covering $C_{l^{n}} \rightarrow \mathcal{M}$ factors through the quotient $\pi_{1}(\mathcal{M}) \rightarrow$ $\Phi^{\{l\}}$. Moreover, to verify this fact, it follows immediately from [3, Proposition 25, (i)] in the case where we take " $(X, Y, n)$ " to be $\left(\mathcal{M}, C_{l^{n}}, 1\right)$ that it suffices to verify that the kernel of the pro-l outer Galois representation associated to $\mathcal{M} / k$ (i.e., $\left.\operatorname{Ker}\left(\rho^{\{l\}}\right)\right)$ coincides with the kernel of the pro- $l$ outer Galois representation associated to $C_{l^{n}} / k$. On the other hand, this follows immediately from [3, Proposition 29], together with Proposition 2.2, (i), (iii); Remark 2.3. This completes the proof of the assertion (ii).

Proposition 2.6. Let $l$ be a prime number and $\lambda \in k \backslash\{0,1\}$. Suppose that one of the following conditions is satisfied:

(1) $\quad l$ is odd, and, moreover, there exist $\lambda_{0} \in \boldsymbol{E}_{l} \cap k$ and a root of unity $u \in k$ such that $\lambda=u \lambda_{0}$.

(2) $\lambda$ is a root of unity.

Then the split hyperbolic curve of type $(0,4)$ over $k$

$$
\boldsymbol{P}_{k}^{1} \backslash\{0,1, \lambda, \infty\}
$$

is not quasi-l-monodromically full.

PROOF. To verify Proposition 2.6, it follows immediately from Lemma 1.3, together with the exactness of the sequence appearing in Definition 1.1, (iii), that, by replacing $k$ by $k^{\text {tpd-l}} \subseteq \bar{k}$ (cf. Definition 1.1, (iv)), we may assume without loss of generality that $k=k^{\text {tpd- } l}$. Write $\phi$ for the composite

$$
G_{k} \stackrel{s_{\lambda}}{\longrightarrow} \pi_{1}(\mathcal{M}) \longrightarrow Q_{l},
$$

where the second arrow is the natural surjection from $\pi_{1}(\mathcal{M})$ to the quotient $Q_{l}$ defined in the statement of Lemma 2.5 (cf. Proposition 2.2, (i), (iii), together with Remark 2.3). Moreover, for each positive integer $n$, write $\phi_{n}$ for the composite of $\phi$ and the natural surjection $Q_{l} \rightarrow$ $Q_{l} / l^{n} Q_{l}\left(\simeq\left(\boldsymbol{Z} / l^{n} \boldsymbol{Z}\right)^{\oplus 2}\right)$ and $k_{n} \subseteq \bar{k}$ for the finite Galois extension of $k$ corresponding to the quotient of $G_{k}$ determined by the homomorphism $\phi_{n}$. Thus, it follows from Lemma 2.5, (i), that

$$
k_{n}=k\left(\lambda^{1 / l^{n}},(1-\lambda)^{1 / l^{n}}\right) .
$$

Now we claim that for any positive integer $n$, it holds that $k_{n}=k\left((1-\lambda)^{1 / l^{n}}\right)$. Indeed, write $u \stackrel{\text { def }}{=} \lambda$ if the condition (2) is satisfied. (Thus, $u$ is always a root of unity of $k$.) Then since $u \in k$ is a root of unity, there exists a root of unity $u_{\neq l} \in k\left(\mu_{l} \infty\right)$ whose order is prime to $l$ such that $u \cdot u_{\neq l} \in \mu_{l^{\infty}}$. Now one verifies easily that $u^{1 / l^{n}} \cdot u_{\neq l} \in \mu_{l^{\infty}}$. In particular, it follows immediately from Proposition 2.2, (i), (iii), together with Remark 2.3, that $u^{1 / l^{n}} \in k\left(\mu_{l^{\infty}}\right) \subseteq k^{\text {tpd-l}}=k$. On the other hand, if the condition (1) is satisfied, then since $\lambda_{0} \in \boldsymbol{E}_{l}$, it follows immediately from Proposition 2.2, (i), (ii), that $\lambda_{0}^{1 / l^{n}} \in \boldsymbol{E}_{l} \subseteq k^{\text {tpd-l }}=k$. In particular, it holds that $k_{n}=k\left(\lambda^{1 / l^{n}},(1-\lambda)^{1 / l^{n}}\right)=k\left((1-\lambda)^{1 / l^{n}}\right)$. This completes the proof of the claim. 
Now it follows immediately from Lemma 1.3 that the hyperbolic curve of type $(0,4)$ over $k$

$$
\boldsymbol{P}_{k}^{1} \backslash\{0,1, \lambda, \infty\}
$$

is quasi- $l$-monodromically full if and only if the image of the composite

$$
G_{k} \stackrel{s_{\lambda}}{\longrightarrow} \pi_{1}(\mathcal{M}) \longrightarrow \Phi^{\{l\}}
$$

is an open subgroup of $\Phi^{\{l\}}$. In particular, it follows from Lemma 2.5, (ii), that if $\boldsymbol{P}_{k}^{1} \backslash$ $\{0,1, \lambda, \infty\}$ is quasi- $l$-monodromically full, then the image of $\phi$ is an open subgroup of $Q_{l}$. On the other hand, it follows immediately from the above claim that for any positive integer $n$, the image of $\phi_{n}$ is a cyclic group. In particular, the image of $\phi$ is not open in $Q_{l}$. Therefore, $\boldsymbol{P}_{k}^{1} \backslash\{0,1, \lambda, \infty\}$ is not quasi- $l$-monodromically full. This completes the proof of Proposition 2.6.

The following result is a consequence of [2, Corollary 7.11]. However, for the reader's convenience, a detailed proof will be given.

PROPOSITION 2.7. Suppose that $k$ is a number field. Write $\mathfrak{o}_{k}$ for the ring of integers of $k$. If $\lambda \in k \backslash\{0,1\}$ satisfies the condition that

$$
\left\{\lambda, 1-\lambda, \frac{\lambda}{\lambda-1}\right\} \cap \mathfrak{o}_{k}^{\times}=\emptyset,
$$

then there exists a finite subset $\Sigma$ of $\mathfrak{P r i m e s}$ such that the split hyperbolic curve of type $(0,4)$ over $k$

$$
\boldsymbol{P}_{k}^{1} \backslash\{0,1, \lambda, \infty\}
$$

is (Primes $\backslash \Sigma$ )-monodromically full.

ProOF. For $t \in k \backslash\{0,1\}$, write $\mathfrak{P}(t)$ for the subset of $\mathfrak{P r i m e s}$ consisting of odd prime numbers $l$ such that the following condition is satisfied: There exists a pair $(\mathfrak{p}, \mathfrak{q})$ of nonarchimedean primes of $k$ such that the residue characteristic of $\mathfrak{p}$ (resp. $\mathfrak{q}$ ) is not equal to $l$, and, moreover, if we write $v_{\mathfrak{p}}: k_{\mathfrak{p}}^{\times} \rightarrow \boldsymbol{Z}$ (resp. $v_{\mathfrak{q}}: k_{\mathfrak{q}}^{\times} \rightarrow \boldsymbol{Z}$ ) for the surjective $\mathfrak{p}$-adic (resp. $\mathfrak{q}$-adic) valuation of the $\mathfrak{p}$-adic (resp. q-adic) completion $k_{\mathfrak{p}}$ (resp. $k_{\mathfrak{q}}$ ) of $k$, then $v_{\mathfrak{p}}(t) \notin l \cdot \boldsymbol{Z}$, $v_{\mathfrak{q}}(1-t) \notin l \cdot \boldsymbol{Z}$, and $v_{\mathfrak{q}}(t)=v_{\mathfrak{p}}(1-t)=0$. Note that it follows from [2, Lemma 7.6, (v)], together with [2, Remark 7.7.1], that if

$$
\left\{t, 1-t, \frac{t}{t-1}\right\} \cap \mathfrak{o}_{k}^{\times}=\emptyset,
$$

then there exists an element

$$
t^{\prime} \in\left\{t, 1-t, \frac{t}{t-1}, \frac{1}{t}, \frac{1}{1-t}, \frac{t-1}{t}\right\}
$$

such that $\mathfrak{P r i m e s} \backslash \mathfrak{P}\left(t^{\prime}\right)$ is finite. Thus, since, for such a $t^{\prime}, \boldsymbol{P}_{k}^{1} \backslash\{0,1, t, \infty\}$ is isomorphic to $\boldsymbol{P}_{k}^{1} \backslash\left\{0,1, t^{\prime}, \infty\right\}$ over $k$, to verify Proposition 2.7 , by replacing $\lambda$ by a suitable element if necessary, we may assume without loss of generality that $\mathfrak{P r i m e s} \backslash \mathfrak{P}(\lambda)$ is finite. Thus, to verify Proposition 2.7, it suffices to verify that if $l \in \mathfrak{P}(\lambda)$, then $X \stackrel{\text { def }}{=} \boldsymbol{P}_{k}^{1} \backslash\{0,1, \lambda, \infty\}$ is 
$l$-monodromically full. The rest of the proof of Proposition 2.7 is devoted to verifying that if $l \in \mathfrak{P}(\lambda)$, then $X$ is $l$-monodromically full.

Let $l$ be an odd prime number. Write $\psi$ for the composite of natural surjections

$$
\pi_{1}\left(\mathcal{M} \otimes_{k} k^{\mathrm{tpd}-l}\right) \longrightarrow \Delta^{\{l\}} \longrightarrow\left(\left(\Delta^{\{l\}}\right)^{\mathrm{ab}}\right) \otimes \mathbf{z}_{l} \boldsymbol{F}_{l} \simeq \boldsymbol{F}_{l}^{\oplus 2},
$$

where the first arrow is the surjection induced by the natural surjection $\pi_{1}(\mathcal{M}) \rightarrow \Phi^{\{l\}}$ (cf. Definition 1.1, (iii)). Since the composite

$$
\operatorname{Gal}\left(\bar{k} / k^{\mathrm{tpd}-l}\right) \stackrel{s_{\lambda}}{\longrightarrow} \pi_{1}\left(\mathcal{M} \otimes_{k} k^{\mathrm{tpd}-l}\right) \longrightarrow \Delta^{\{l\}},
$$

where the second arrow is the surjection induced by the natural surjection $\pi_{1}(\mathcal{M}) \rightarrow \Phi^{\{l\}}$, is surjective if and only if the composite

$$
\operatorname{Gal}\left(\bar{k} / k^{\mathrm{tpd}-l}\right) \stackrel{s_{\lambda}}{\longrightarrow} \pi_{1}\left(\mathcal{M} \otimes_{k} k^{\mathrm{tpd}-l}\right) \stackrel{\psi}{\longrightarrow}\left(\left(\Delta^{\{l\}}\right)^{\mathrm{ab}}\right) \otimes_{\mathbf{Z}_{l}} \boldsymbol{F}_{l} \simeq \boldsymbol{F}_{l}^{\oplus 2}
$$

is surjective (cf., e.g., [5, Corollary 2.8.5], together with [5, Lemma 2.8.7, (c)]), it follows immediately from Lemma 1.3 that, to verify that $X$ is $l$-monodromically full, it suffices to verify that the Galois group over $k^{\text {tpd-l}}$ of the finite Galois extension of $k^{\text {tpd-l }}$ corresponding to the quotient of $\mathrm{Gal}\left(\bar{k} / k^{\mathrm{tpd}-l}\right)$ determined by the composite

$$
\operatorname{Gal}\left(\bar{k} / k^{\mathrm{tpd}-l}\right) \stackrel{s_{\lambda}}{\longrightarrow} \pi_{1}\left(\mathcal{M} \otimes_{k} k^{\mathrm{tpd}-l}\right) \stackrel{\psi}{\longrightarrow}\left(\left(\Delta^{\{l\}}\right)^{\mathrm{ab}}\right) \otimes_{\mathbf{Z}_{l}} \boldsymbol{F}_{l} \simeq \boldsymbol{F}_{l}^{\oplus 2}
$$

is isomorphic to $\boldsymbol{F}_{l}^{\oplus 2}$. On the other hand, it follows immediately from Lemma 2.5, (ii), together with the well-known structure of the abelianization of $\Delta^{\{l\}}$, that the finite étale Galois $\boldsymbol{F}_{l}^{\oplus 2}$-covering of $\mathcal{M} \otimes_{k} k^{\text {tpd-l}}$ corresponding to the surjection $\psi$ is the finite étale covering

$$
C_{l}^{\mathrm{tpd}-l} \stackrel{\text { def }}{=} \operatorname{Spec} k^{\mathrm{tpd}-l}\left[x^{ \pm 1}, y^{ \pm 1}\right] /\left(x^{l}+y^{l}-1\right) \longrightarrow \mathcal{M} \otimes_{k} k^{\mathrm{tpd}-l},
$$

where $x$ and $y$ are indeterminates, given by " $t \mapsto x^{l}$ ". Thus, by Lemma 2.5, (i), we conclude that, to verify that $X$ is $l$-monodromically full, it suffices to verify that

$$
\operatorname{Gal}\left(k^{\mathrm{tpd}-l}\left(\lambda^{1 / l},(1-\lambda)^{1 / l}\right) / k^{\mathrm{tpd}-l}\right) \simeq \boldsymbol{F}_{l}^{\oplus 2} .
$$

Suppose that $l \in \mathfrak{P}(\lambda)$. Let $\zeta_{l} \in k^{\text {tpd- } l}$ be a primitive $l$-th root of unity (cf. Proposition 2.2, (i), (iii)). Now since $l \in \mathfrak{P}(\lambda)$, and $\left[k\left(\zeta_{l}\right): k\right]<l$, it follows immediately that there exists a pair $(\mathfrak{p}, \mathfrak{q})$ of nonarchimedean primes of $k\left(\zeta_{l}\right)$ such that the residue characteristic of $\mathfrak{p}$ (resp. $\mathfrak{q}$ ) is not equal to $l$, and, moreover, $v_{\mathfrak{p}}(\lambda) \notin l \cdot \boldsymbol{Z}, v_{\mathfrak{q}}(1-\lambda) \notin l \cdot \boldsymbol{Z}$, and $v_{\mathfrak{q}}(\lambda)=v_{\mathfrak{p}}(1-\lambda)=0$. Then one verifies easily that the finite Galois extension $k\left(\zeta_{l}, \lambda^{1 / l}\right) / k\left(\zeta_{l}\right)\left(\right.$ resp. $\left.k\left(\zeta_{l},(1-\lambda)^{1 / l}\right) / k\left(\zeta_{l}\right)\right)$ is [necessarily totally tamely] ramified at $\mathfrak{p}$ (resp. q) and unramified at $\mathfrak{q}$ (resp. p). Therefore, it follows immediately from Proposition 2.2, (iv), that the Galois group $\operatorname{Gal}\left(k^{\text {tpd-l}}\left(\lambda^{1 / l},(1-\lambda)^{1 / l}\right) / k^{\text {tpd-l }}\right)$ is isomorphic to $\boldsymbol{F}_{l}^{\oplus 2}$. This completes the proof of Proposition 2.7 . 
3. Proofs of main results. In the present section, we give proofs of Theorems A, B in Introduction.

Proof of Theorem A. Let $l$ be an odd prime number. Then since $l \in \boldsymbol{E}_{l}$ (cf. Lemma 2.4), it follows immediately from Proposition 2.6 that the hyperbolic curve of type $(0,4)$ over $k$

$$
X \stackrel{\text { def }}{=} \boldsymbol{P}_{k}^{1} \backslash\{0,1, l, \infty\}
$$

is not quasi- $l$-monodromically full. On the other hand, since neither $l, 1-l$, nor $l /(l-1)$ is a unit of the ring of integers of $k$, it follows from Proposition 2.7 that there exists a finite subset $\Sigma$ of $\mathfrak{P r i m e s}$ such that $X$ is (Primes $\backslash \Sigma$ )-monodromically full. In particular, $X$ satisfies the condition $\left(\mathrm{MT}_{3}\right)$ but does not satisfy the condition $\left(\mathrm{MT}_{1}\right)$. This completes the proof of the fact that there exists a split hyperbolic curve of type $(0,4)$ over $k$ which satisfies $\left(\mathrm{MT}_{3}\right)$, hence also $\left(\mathrm{MT}_{2}\right)$, but does not satisfy $\left(\mathrm{MT}_{1}\right)$.

Moreover, let $r>4$ be a positive integer and $l^{\prime} \in \mathfrak{P r i m e s} \backslash \Sigma$ (cf. the notation of the preceding paragraph). Then it follows from [3, Proposition 13] in the case where we take " $(X, n)$ " to be $(X, r-4)$ that there exists an $l^{\prime}$-monodromically full $k$-rational point $x$ (cf. [3, Definition 8, (i)] in the case where we take " $(X, n)$ " to be $(X, r-4))$ of the $(r-4)$-th configuration space of the hyperbolic curve $X / k$. Since $X$ is $l^{\prime}$-monodromically full and $x$ is $l^{\prime}$-monodromically full, it follows from [3, Proposition 21] in the case where we take " $(X, n)$ " to be $(X, r-4)$ that the split hyperbolic curve $Y$ of type $(0, r)$ determined by $x$, i.e., the hyperbolic curve obtained by taking the complement in $X$ of the images of $r-4$ distinct $k$ rational points of $X$ determined by $x$, is $l^{\prime}$-monodromically full. In particular, $Y$ satisfies the condition $\left(\mathrm{MT}_{2}\right)$. On the other hand, since $Y \subseteq X$, and $X$ is not quasi- $l$-monodromically full, it follows from [2, Remark 2.2.5] that $Y$ is not quasi- $l$-monodromically full. In particular, $Y$ does not satisfy the condition $\left(\mathrm{MT}_{1}\right)$. This completes the proof of the fact that for any positive integer $r>4$, there exists a split hyperbolic curve of type $(0, r)$ over $k$ which satisfies $\left(\mathrm{MT}_{2}\right)$ but does not satisfy $\left(\mathrm{MT}_{1}\right)$.

Proof of TheOREM B. First, let us observe that it follows immediately from the various definitions involved that, to verify the implication "(1) $\Rightarrow(2)$ ", by replacing $X / k_{0}$ by replacing $X \otimes_{k_{0}} k / k$, we may assume without loss of generality that $X$ is split over $k_{0}$. Then the implication "(1) $\Rightarrow$ (2)" follows from Proposition 2.7. The implications "(2) $\Rightarrow(3) \Rightarrow(4)$ " are immediate. The implication "(4) $\Rightarrow(5)$ " follows immediately from Proposition 2.6, together with [2, Remark 2.2.6]. The implication " $(5) \Rightarrow(1)$ " in the case where $k$ is imaginary quadratic follows immediately from the well-known fact that every unit of the ring of integers of an imaginary quadratic field is a root of unity.

\section{REFERENCES}

[ 1 ] G. Anderson And Y. Ihara, Pro- $l$ branched coverings of $\boldsymbol{P}^{1}$ and higher circular $l$-units, Ann. of Math. (2) 128 (1988), 271-293.

[2] Y. HoshI, Galois-theoretic characterization of isomorphism classes of monodromically full hyperbolic curves of genus zero, Nagoya Math. J. 203 (2011), 47-100. 
[3] Y. Hoshi, On monodromically full points of configuration spaces of hyperbolic curves, The Arithmetic of Fundamental Groups-PIA 2010, 167-207, Contributions in Mathematical and Computational Sciences, vol. 2, Springer-Verlag, Berlin, Heidelberg, 2012.

[ 4 ] M. Matsumoto And A. Tamagawa, Mapping-class-group action versus Galois action on profinite fundamental groups, Amer. J. Math. 122 (2000), 1017-1026.

[ 5 ] L. Ribes AND P. ZALESS KII, Profinite groups, Ergebnisse der Mathematik und ihrer Grenzgebiete, 3, Folge, A Series of Modern Surveys in Mathematics, 40, Springer-Verlag, Berlin, 2000.

[6] J. P. SERre, Propriétés galoisiennes des points d'ordre fini des courbes elliptiques, Invent. Math. 15 (1972), 259-331.

RESEARCH INSTITUTE FOR MATHEMATICAL SCIENCES

KYOTO UNIVERSITY

KYOTO 606-8502

JAPAN

E-mail address: yuichiro@kurims.kyoto-u.ac.jp 